\title{
Dynamic Change of Cellular Localization of Microtubule- Organizing Center During Conjugation of Ciliate Tetrahymena thermophila
}

\author{
Yasuharu Kushida, Masak Takaine, Kentaro Nakano, \\ Toshiro Sugai, and Osamu Numata* \\ Graduate School of Life and Environmental Sciences, University of Tsukuba, \\ 1-1-1 Tennodai, Tsukuba, Ibaraki 305-8572, Japan
}

\begin{abstract}
To obtain a comprehensive picture of microtubule dynamics during conjugation, the mode of sexual reproduction in ciliates, we combined indirect immunofluorescence and three-dimensional imaging using confocal laser-scanning microscope to visualize the cellular localization of DNA, microtubules, and $\gamma$-tubulin, the main component of the microtubule-organizing center in mating Tetrahymena cells. As the conjugational stages proceeded, the distribution of $\gamma$-tubulin changed drastically and microtubules showed dynamic appearance and disappearance during meiosis, nuclear selection, nuclear exchange, and the development of new macronuclei. This study highlights the involvement of cytoskeletal regulation in the modulation of germline nuclear motilities required for ciliate reproduction.
\end{abstract}

Key words: ciliates, conjugation, $\gamma$-tubulin, microtubule, Tetrahymena

\section{INTRODUCTION}

Ciliate cells contain two functionally differentiated nuclei. Polyploid macronucleus (MAC) actively expresses all necessary genes for proliferation of the cell, and diploid micronucleus (MIC) stores intact chromosomes for sexual reproduction (Allen and Gibson, 1972). During the developmental process of ciliate, called conjugation, a new MAC is differentiated from a germline MIC, and the parental old MAC is degraded, enabling the successful alternation of generations.

The microtubule (MT) structures of ciliate cells are well developed. Tetrahymena cell has 17 kinds of MT structures including cilia, cortical MTs underlying cell surface, intranuclear MTs in dividing nucleus, and cytoplasmic MTs bridging the gap between organelles and the cell surface (Gaertig, 2000). Especially, cortical MTs are important to determine the polarity of the cell (Wloga and Frankel, 2012). To preserve the cortical structure during conjugation, two mating ciliate cells never fuse completely. Instead, two ciliate cells form a partial protoplasmic connection (conjugation junction) for the subsequent genetic crossing (Cole, 2006; Cole and Sugai, 2012). After meiosis, additional nuclear division called gametogenic mitosis produce two pronuclei, and one of them will transport across conjugation junction for the exchange of pronuclei between partner cells. Fertilized nucleus divides unequally to develop new MAC and new MIC during post-zygotic nuclear divisions. At the completion of conjugation, two mating cells separate and start growing

\footnotetext{
* Corresponding author. Tel. : : +81-29-853-6648;

Fax : +81-29-853-6614;

E-mail: numata.osamu.gb@u.tsukuba.ac.jp Supplemental material for this article is available online. doi:10.2108/zs140149
}

as independent progenies.

Treatment with MT-depolymerizing drugs has shown that MT structures are involved in almost all processes during conjugation (Kaczanowski et al., 1985, 1991; Hamilton et al., 1988). Electron microscopy (EM) demonstrated the formation of MT bundles during prophase of meiosis (Wolfe et al., 1976; Suganuma and Yamamoto, 1992). In a later study using indirect immunofluorescence, Gaertig and Fleury (1992) succeeded in obtaining a general picture of MT distribution during conjugation of Tetrahymena.

Interestingly, the germline nucleus locates at a stagespecific position of the cell during Tetrahymena conjugation (Cole and Sugai, 2012). In fertilized egg, both male and female pronuclei approach the sperm centrosome by MTmotor-driven movement along centrosomal astral MTs, yielding a fertilized nucleus. This mechanism is conserved from Caenorhabditis elegans (Gönczy et al., 1999; Malone et al., 2003; Minn et al., 2009) to vertebrate (Lindeman and Pelegri, 2012). The movement of Tetrahymena germline pronuclei seems to depend on the polarity of cortical structures instead of centrosome of animal cells. Moreover, the position of pronuclei changes drastically corresponding to the progression of conjugational stage. This suggests the underlying mechanism placing the nuclei at particular area in a particular stage of conjugation. Because the Tetrahymena conjugational stage alternates quickly in an MT-dependent way, it is tempting to assume that rapid qualitative changes in MTs corresponding to cell polarity occur throughout the conjugational process in mating Tetrahymena cells.

Previously, we analyzed a localization of $\gamma$-tubulin, a key component of MT-organizing center (MTOC) in Tetrahymena cell, to address MT-formation in dividing MAC during vegetative growth. By using deconvolution processing, we 
obtained low-background three-dimensional images of intraMAC MTs and succeeded to reveal the dynamic change of distribution of MT and MTOC during amitotic MAC division (Kushida et al., 2011).

Here we use indirect immunofluorescence and confocal laser-scanning microscopy to show three-dimensional distribution of MT and MTOC during Tetrahymena conjugation. The localization analysis of MT and MTOC in conjugating Tetrahymena sheds light on the dynamic property of MT structures during conjugation of ciliate cells.

\section{MATERIALS AND METHODS}

\section{Cell strain and media}

To investigate the distribution of $\gamma$-tubulin during conjugation of Tetrahymena, strain CU428 was crossed with strain cTTMGHA (Shang et al., 2002), which expresses HA-tagged Tetrahymena $\gamma$-tubulin under the cadmium-ion inducible promoter (MTT1). The strain CTTMGHA and the strain CU428 were kindly donated by Prof. Martin A. Gorovsky (University of Rochester) and Prof. Jacek Gaertig (University of Georgia), respectively. Both strains were cultured in SPP medium (1\% (w/v) proteose peptone, $0.1 \%(\mathrm{w} / \mathrm{v})$ yeast extract, $0.2 \%(\mathrm{w} / \mathrm{v})$ D-glucose, $0.003 \%(\mathrm{w} / \mathrm{v})$ $\mathrm{Fe}$ (III)-EDTA). One microgram per milliliter of $\mathrm{CdCl}_{2}$ was added to the CTTMGHA culture (Shang et al., 2002). The cells were starved in $10 \mathrm{mM}$ Tris$\mathrm{HCl}\left(\mathrm{pH} \mathrm{7.5)}\right.$ at $30^{\circ} \mathrm{C}$ overnight before mating.

\section{Fluorescence microscopy}

To induce conjugation, equal numbers $\left(\sim 10^{5}\right.$ cells $/ \mathrm{mL}$ ) of starved cells from each strain were mixed and incubated at $30^{\circ} \mathrm{C}$. The conjugation between CTTMGHA and CU428 (wildtype) proceeds normally, and produces new MAC and new MIC as in wildtype-wildtype cross. Cells were sampled from 1.5 to $9.5 \mathrm{~h}$ at intervals of $15 \mathrm{~min}$ and fixed in accordance with the methods of Kushida et al. (2011). Anti- $\alpha$-tubulin antibody and anti-HA antibody were used to detect MTs and a HA-tagged recombinant $\gamma$-tubulin (GTU1-HA) expressed in CTTMGHA, respectively. Fixed cells were treated with mouse anti-chicken $\alpha$-tubulin antibody (Calbiochem, Darmstadt, Germany; 1/300 dilution) and rabbit polyclonal anti-HA antibody (Bethyl Laboratories, Montgomery, TX; 1/300 dilution) at room temperature overnight. After being washed, the cells were incubated with FITC-conjugated goat anti-mouse IgG antiserum (TAGO, Burlingame, CA; 1/300 dilution) and rhodamine-conjugated goat antirabbit IgG antiserum (Kirkegaard \& Perry Laboratories, Gaithersburg, MD; $1 / 300$ dilution) for $6 \mathrm{~h}$ at room temperature. DNA was stained with $4^{\prime}, 6-$ diamidino-2-phenylindole (DAPI; final concentration, $1 \mu \mathrm{g} / \mathrm{mL}$ ). These cells were immobilized between a slide glass and the cover slip, which was tilted by asymmetric insertion of a spacer to prevent the cell from being flattened. Cells were observed under a confocal laser scanning microscope (Carl Zeiss, Oberkochen, Germany, LSM700) with an alpha Plan-Apochromat $100 \times$ lens with a numerical aperture of 1.46 . The cells positioned in optimal range of space (thickness of about $12-20 \mu \mathrm{m}$ ) between coverslip and slide glass were imaged. The conjugational stages were identified by refer- ring to previous studies (Gaertig and Fleury, 1992; Cole and Sugai, 2012). Since ciliate cell have massive fluorescent signals on cell cortex (see Supplementary Figure S1 online; Fig. S1A, left panel), z-stacking was performed to eliminate unnecessary cortical signals (Fig. S1A, right panel). The brightness and contrast of images was adjusted manually by Adobe Photoshop or Image $\mathrm{J}$.
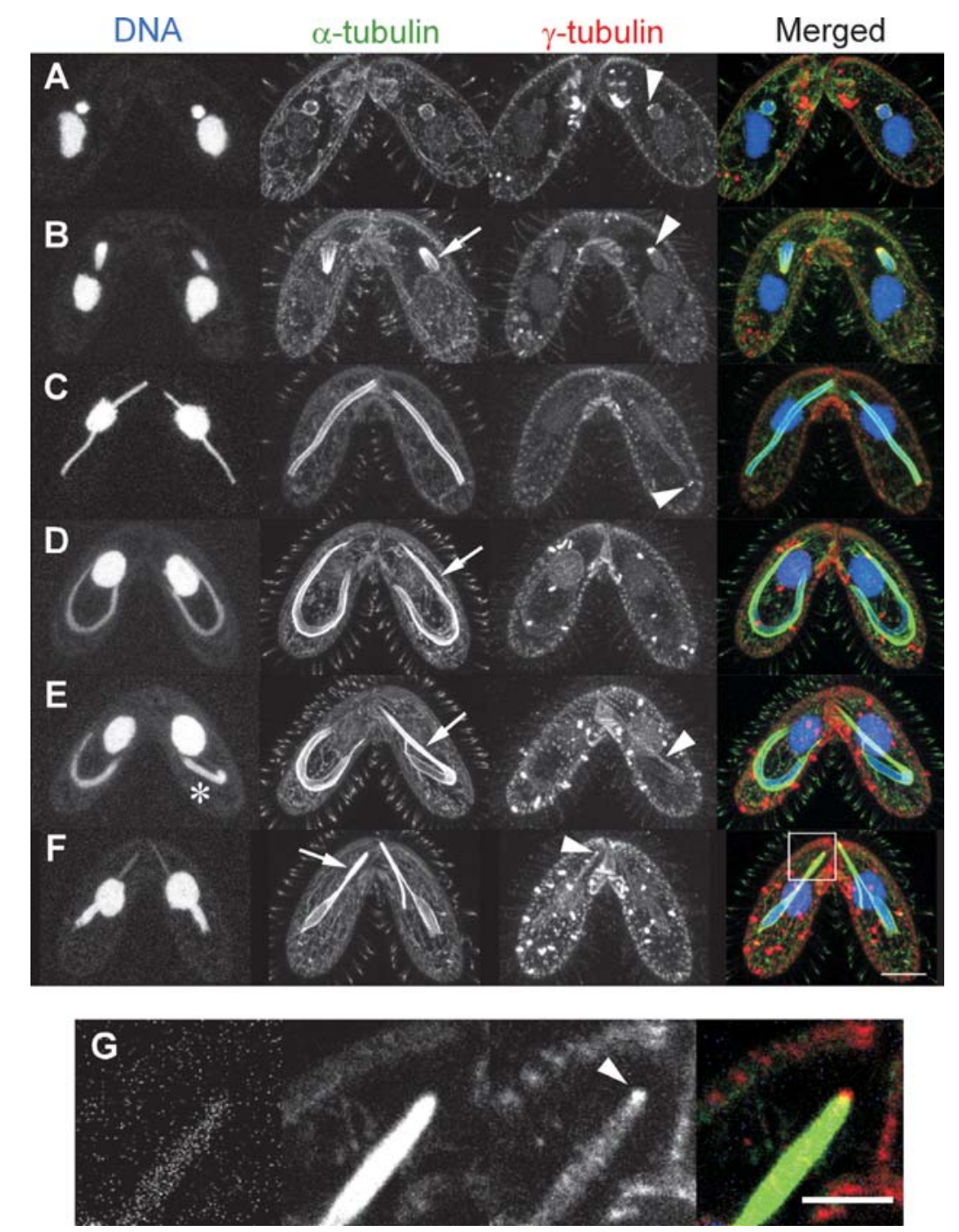

Fig. 1. Localization of DNA, MTs and $\gamma$-tubulin during elongation and shortening of crescent. Four sets of Z-stack images of each row were obtained from the same pair of cells in the same focal plane. (A) Early prophase of meiosis I. The signal of $\gamma$-tubulin is detectable (arrowhead). (B) The beginning of elongation of crescent. $\gamma$-tubulin was accumulated on one end of crescent (arrowhead) and MTs were polymerized from there (arrow). (C) Partially elongated crescent. The accumulation of $\gamma$-tubulin was decreased compared to previous stage (arrowhead). (D) Full-length crescent. MT bundles become about twice as long as cell body (arrow). $\gamma$-tubulin is dispersed along crescent (right panel). Some bright granule-like signals, which did not colocalize with MT, were detected in cytoplasm until about the end of meiosis I (see Fig. 2). (E) The beginning of the shortening of crescent. When chromosome condense (asterisk), MTs started to bundle together (arrow) and $\gamma$-tubulin was accumulated on the thick MT bundles. (F) Partially shortened crescent. $\gamma$-tubulin (arrowhead) was clearly accumulated at the end of MT bundle (arrow). Scale bar (A-F): $10 \mu \mathrm{m}$. (G) Enlarged image of the boxed area in $(F)$. Single focus image is shown. $\gamma$-tubulin accumulated the edge of MT bundle. Scale bar (G): $4 \mu \mathrm{m}$. 


\section{RESULTS}

\section{$\gamma$-tubulin is accumulated on one end of MIC at the start of elongation of 'crescent'}

For the purpose of localization analysis of $\gamma$-tubulin, a transgenic mutant which overproduce HA-tagged $\gamma$-tubulin (strain cTTMGHA) was mated with wildtype cell (strain CU428), and the mating pair was processed for triple staining of DNA, MT and $\gamma$-tubulin by immunofluorescence. In the wildtype cell, the fluorescent signal of $\gamma$-tubulin was detectable during early prophase of meiosis I, probably due to the diffusion of HA-tagged $\gamma$-tubulin from mutant cell to wildtype cell through conjugation junction. The pair of $\gamma$-tubulin-overproducing cell and wildtype cell normally completed the conjugational process and developed a new MAC. Since the MT distribution of mutant-wildtype pair was similar to that of wildtype-wildtype pair (Fig. S1), further description is based on data obtained from observation of mutant-wildtype pairs.

When the MIC was detached from the MAC, several dotted fluorescent signals of $\gamma$-tubulin were detected on the surface of the MIC (Fig. 1A, arrowhead, $n=14$ ). In Tetrahymena, the pairing of homologous chromosomes occurs in extremely elongated MIC (the "crescents"; Loidl and Scherthan, 2004; Loidl and Mochizuki, 2009; Loidl et al., 2012). At the beginning of crescent elongation, $\gamma$-tubulin was strongly accumulated at one end of the MIC (Fig. 1B, arrowhead, $n=29$ ) and may rapidly produce a large number of MTs in one direction (Fig. 1B, arrow). During expansion of the crescent, a layer of MTs is observed using EM (Wolfe et al., 1976; Suganuma and Yamamoto, 1992). This group of MTs possibly produces force required for the crescent elongation.

\section{$\gamma$-tubulin dispersed over fully-elongated MIC 'crescent'}

During the elongation of crescent, most of $\gamma$-tubulin dots dispersed on MT bundles, while some $\gamma$-tubulin dots stayed at the one end of the crescent (Fig. 1C, arrowhead, $n=16$ ). When the crescent completes elongation, its length was about two times of the length of the cell body (Fig. 1D, arrow). At that time, $\gamma$ tubulin dots distributed uniformly along full length of the crescent, and all $\gamma$-tubulin was released from one end of crescent (Fig. 1D, right panel, $n=14)$. In the photo of $\gamma$-tubulin, some bright granule-like signals were detected in cytoplasm about from the crescent (prophase) to anaphase of meiosis I (Fig. 1D-F, 2B-D), which did not colocalize with MTs. Since the distribution pattern of the granule-like signals were indefinite and seemed not to be MTOC, we exclude them from further examination.

\section{$\gamma$-tubulin dots accumulated at both ends of the shortening crescent}

After crescent fully elongates and MIC chromosomes finish homologous pairing, the chromosomes start condensing toward one side of the crescent (Fig. 1E, asterisk). MT bundles positioned in chromosome-free region of the crescent rapidly coalesced to form thicker MT bundles, and the shortening of the crescent started (Fig. 1E, arrow). These thick MT bundles became thicker and shorter. The dotted signals of $\gamma$-tubulin became obvious on this thick bundle of MTs (Fig. 1E, arrowhead, $n=9$ ). As shortening of the crescent proceeds, $\gamma$-tubulin accumulated on the end of the MT bundle (Fig. 1F, G, arrowheads, $n=7$ ), namely the end of the shortening crescent. Accumulation of $\gamma$-tubulin is also observed on the other end of crescent MTs when the length of the crescent become close to that of the meiotic spindle (Fig. 2A, arrowheads, $n=18$ ). Finally, all the MTs were gathered within about one-third of length of the cell body to form a meiotic spindle. $\gamma$-tubulin was clearly detected on both ends of the thick MT bundles (Fig. 2B, arrowheads, $n=13$ ). The dotted signals of $\gamma$-tubulin were detected on daughter nuclei of meiosis I (Fig. 2C, D, arrowheads, $n=7,6$, respectively), and $\gamma$-tubulin accumulated again on the spindle poles of meiosis II (Fig. 2E, arrowheads, $n=19$ ).

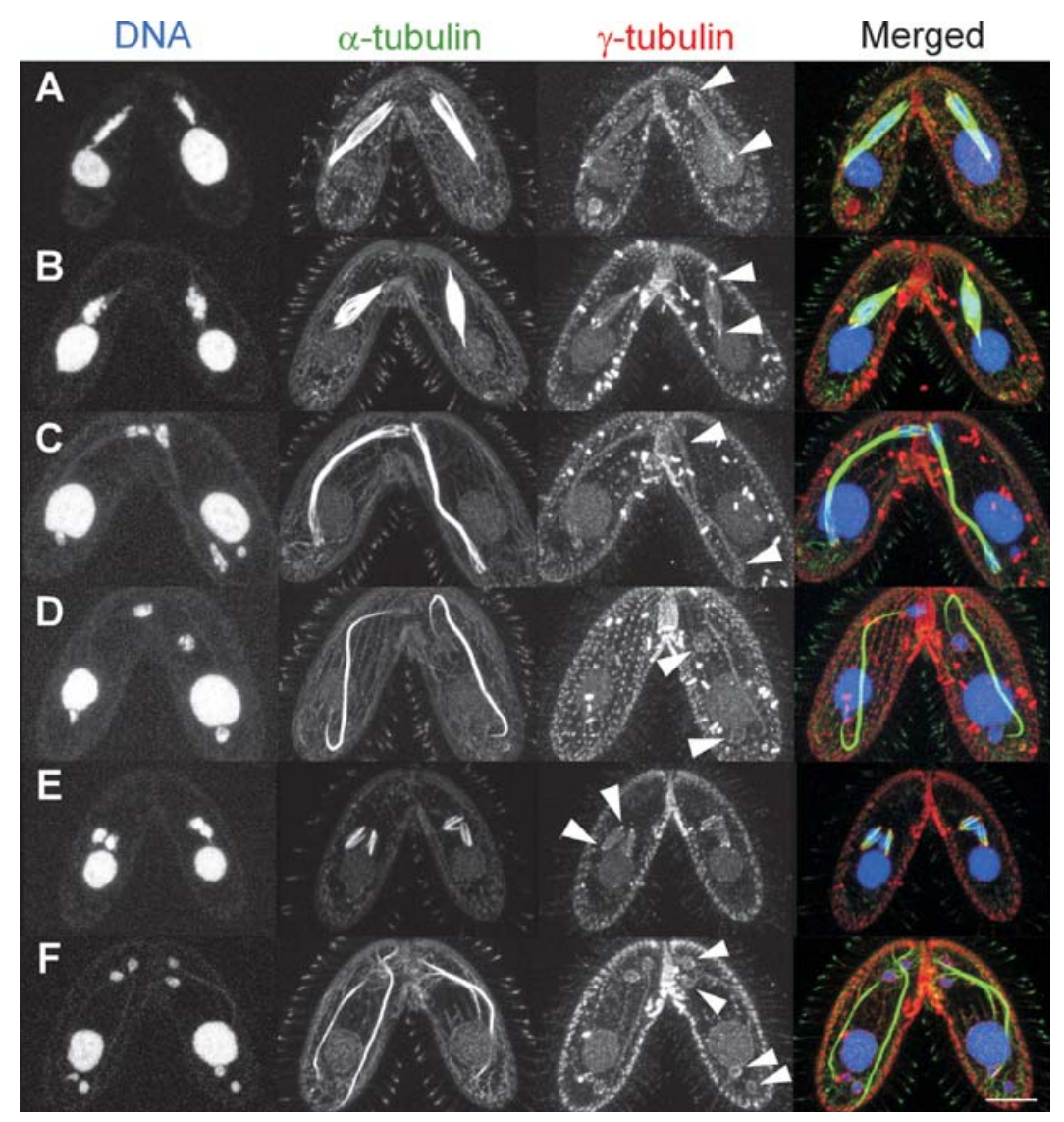

Fig. 2. Localization of DNA, MTs and $\gamma$-tubulin during meiosis I and II. (A) The late period of crescent shortening. $\gamma$-tubulin can be clearly detected on both ends of bundled MTs (arrowheads). (B) Metaphase spindle of meiosis I. $\gamma$-tubulin was accumulated on both spindle poles (arrowheads). (C, D) Anaphase of meiosis I. $\gamma$-tubulin is detectable on both daughter nuclei (arrowheads). (E) Metaphase of meiosis II. As in meiosis I, $\gamma$-tubulin localized on spindle poles. (F) Anaphase of meiosis II. $\gamma$-tubulin was detectable around four daughter nuclei (arrowheads). Scale bar (A-F): $10 \mu \mathrm{m}$. 


\section{Abundant $\gamma$-tubulin accumulated on the surface of the selected post-meiotic nucleus}

Among the four nuclei produced by meiosis (Fig. 2F, arrowheads, $n=13$ ), only one nucleus becomes closely attached to the conjugation junction positioned at the anterior part of the cell (Fig. 3A, large arrowheads). This phenomenon has been termed nuclear "selection" (Cole and Sugai, 2012). At the time of nuclear selection, $\gamma$-tubulin coated the surface of the selected nucleus (Fig. 3A, large arrowheads, $n=10$ ), and radial MTs extended from the selected nucleus to the cytoplasm (Fig. 3A, arrows), suggesting that $\gamma$-tubulin promoted polymerization of the radial MTs. Notably, many $\gamma$-tubulin signals accumulated at the conjugation junction during nuclear selection (Fig. 3A, small arrowhead). The selected gametic nucleus and its derivatives were constantly coated with abundant $\gamma$-tubulin during the later stages of conjugation, namely gametogenic mitosis,
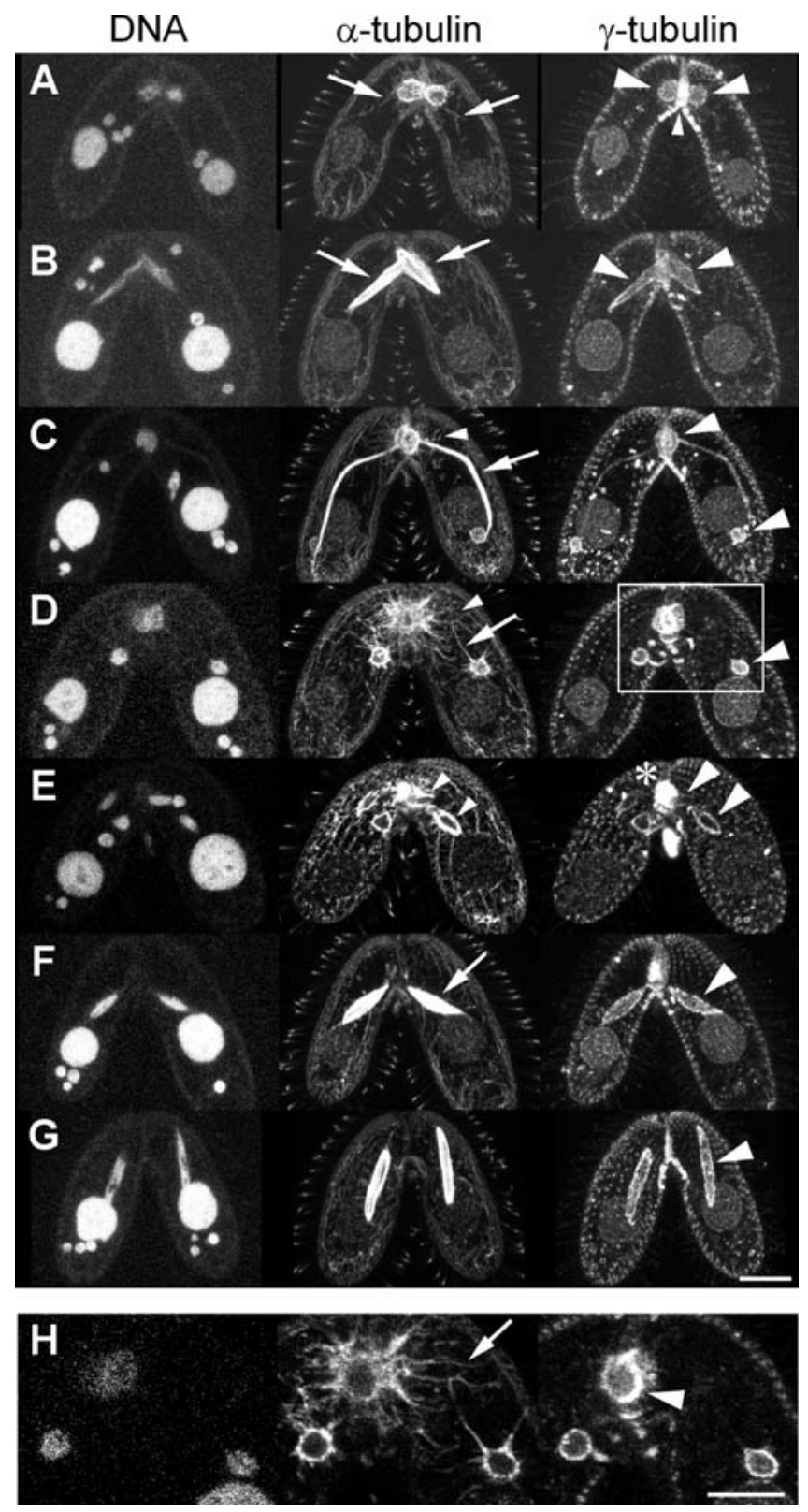

pronuclear migration, pronuclear exchange, fertilization, and post-zygotic division (Fig. 3B-H, 4A, B, large arrowheads).

\section{Radial MTs formed from the migrating nuclei coated with $\gamma$-tubulin}

Two gametic pronuclei are formed by mitosis of the selected post-meiotic nucleus, in a process called gametogenic mitosis (Cole and Sugai, 2012; Fig. 3B, arrows, $n=$ 15). We observed that, during anaphase of gametogenic mitosis, $\gamma$-tubulin accumulated strongly on both daughter pronuclei (Fig. 3C, large arrowheads, $n=8$ ). Whereas the majority of the fluorescent signal from MTs labeled the mitotic spindle that connected the daughter pronuclei (Fig. $3 \mathrm{C}$, arrow), some radial cytoplasmic MTs were observed around the anterior daughter pronucleus (Fig. 3C, small arrowhead). The posterior daughter pronucleus was easily distinguished from the remaining, unselected, post-meiotic nuclei because the pronucleus was strongly labeled with $\gamma$ tubulin (Fig. 3C, lower large arrowhead).

After being separated from the spindle MTs, the posterior pronucleus starts to move to the conjugation junction of the cell, where fertilization occurs (Ray, 1956; Martindale et al., 1982; Cole and Sugai, 2012; Fig. 3D, large arrowhead). We observed that, when the spindle MTs disappeared, radial cytoplasmic MTs were likely to be produced from the $\gamma$-tubulin surrounding the migrating posterior pronucleus (Fig. 3D, arrow, $n=2$ ), as well as from the anterior pronucleus (Fig. 3D, small arrowhead).

During pronuclear migration, the anterior pronucleus is exchanged with the pronucleus of the partner cell. EM studies report that an MT "basket" seems to push the pronucleus against the conjugational junction to enable successful

Fig. 3. Localization of DNA, MTs, and $\gamma$-tubulin during gametogenic mitosis, pronuclear migration, pronuclear exchange, and fertilization. In all panels, $\alpha$-tubulin was used as marker for MTs. The three Z-stack images in each panel $(\mathbf{A}-\mathbf{H})$, respectively, were obtained from the same pairs of cells in the same focal plane. (A) Nuclear selection. $\gamma$-tubulin was selectively accumulated on the single post-meiotic nucleus attached to the cell junction (large arrowheads), and radial MTs originated from the same post-meiotic nucleus (arrows). Also, $\gamma$-tubulin rapidly accumulated on the conjugation junction (small arrowhead). (B) Early anaphase of gametogenic mitosis. $\gamma$-tubulin (arrowheads) distributed across all spindleshaped MTs (arrows). (C) Late anaphase of gametogenic mitosis. $\gamma$ tubulin accumulated on both of the daughter pronuclei (arrowheads) while they were connected by the spindle (arrow). Radial MTs were polymerized from the anterior pronuclei (small arrowhead). (D) Pronuclear exchange. Radial MTs (arrow) were polymerized from the posterior pronucleus (arrowhead) migrating to the anterior side. MTs from the anterior pronucleus are indicated by the small arrowhead. (E) After pronuclear exchange. $\gamma$-tubulin was distributed on both the pronuclei (arrowheads) and the cell junction (asterisk). MTs around the pronuclei are indicated by small arrowheads. (F) Fertilization. The entire spindle-shaped zygotic nucleus (arrow) was labeled with $\gamma$-tubulin (arrowhead). (G) Early anaphase of the first post-zygotic division. $\gamma$-tubulin (arrowhead) localized over the spindle-shaped MTs. Scale bar (A-G): $10 \mu \mathrm{m}$. (H) Enlarged image of marked area in (D). Single focus image is shown. $\gamma$-tubulin accumulated on the "basket" (arrowhead) polymerized radial MTs (arrow). Scale bar (H): $8 \mu \mathrm{m}$. 
transfer of the nucleus (Orias et al., 1983; Cole, 2006). We detected abundant $\gamma$-tubulin on this basket (Fig. $3 \mathrm{H}$, arrowhead) and protrusions of abundant radial cytoplasmic MTs from the basket (Fig. 3H, arrow). These results suggest that $\gamma$-tubulin promotes the polymerization of radial cytoplasmic MTs from the MT basket surrounding the anterior pronucleus.

\section{$\gamma$-tubulin coated the fertilized nucleus and daughter nuclei of post-zygotic division}

The posterior pronucleus that arrives at the conjugation junction of the cell fuses with the anterior pronucleus transferred from the partner cell to become one fertilized nucleus (Cole and Sugai, 2012; Fig. 3F, arrow). We detected $\gamma$-tubulin on the transferred anterior pronuclei (Fig. 3E, upper large arrowhead, $n=2$ ) even after nuclear exchange, though most $\gamma$-tubulin was detected at the conjugation junction (Fig. 3E, asterisk). After fertilization, the spindle-shaped fertilized nucleus was labeled strongly with $\gamma$-tubulin (Fig. 3F, arrowhead, $n=5$ ).

The post-zygotic divisions produce two MICs and two new MACs (Cole and Sugai, 2012). We observed that during the first post-zygotic division, $\gamma$-tubulin was located across all the MTs (Fig. 3G, 4A, arrowheads, $n=2$ ) and accumulated on the surface of the daughter nuclei after separated from spindle (Fig. 4B, large arrowheads, $n=4$ ). This pattern of $\gamma$-tubulin localization is similar to that observed at gametogenic mitosis (Fig. 3C, large arrowheads). The presence of abundant $\gamma$-tubulin coating the nuclei is no longer seen after the second post-zygotic division (Fig. 4C, arrowheads, $n=3$ ).

\section{$\gamma$-tubulin inside swelling new MACs}

After the second post-zygotic division, the two anterior daughter nuclei differentiate to form new MACs and the two posterior nuclei become the two new MICs (Cole and Sugai, 2012). We observed that when the MT spindle of the second post-zygotic division disappeared, the network of cytoplasmic MTs was highly augmented (Fig. 4D, middle panel). Some $\gamma$-tubulin molecules were easily detected in the parental MAC (Fig. 4D, arrow, $n=6$ ), and scattered MTs were formed in the parental MAC (Fig. 4D, small arrowhead). These $\gamma$-tubulin molecules and MTs disappeared quickly when the parental MAC started to condense for degradation (Fig. 4E, arrow, $n=2$ ). Several dotted signals of $\gamma$-tubulin were detected in both of the new MACs (Fig. 4D, E, large arrowheads) as well as in both of the new MICs (Fig. 4E, small arrowheads). The $\gamma$-tubulin signals of new MACs increased (Fig. 4F, arrowheads and Fig. 4G, arrows, $n=5$; for merged images, see Supplementary Figure S2 online) and some dotted signals of MTs were detectable in the new MACs when they swelled (Fig. 4G, arrowheads).

\section{DISCUSSION}

$\gamma$-tubulin disperses on MT bundles in MIC during elongation of meiotic crescent

During elongation of meiotic crescent, the distribution of $\gamma$-tubulin well as that of MTs changed drastically. At the beginning of crescent elongation, $\gamma$-tubulin localized at one end of the crescent (Fig. 1B, arrowhead), and MT bundles were likely to be polymerized from this area (Fig. 1B, arrow).
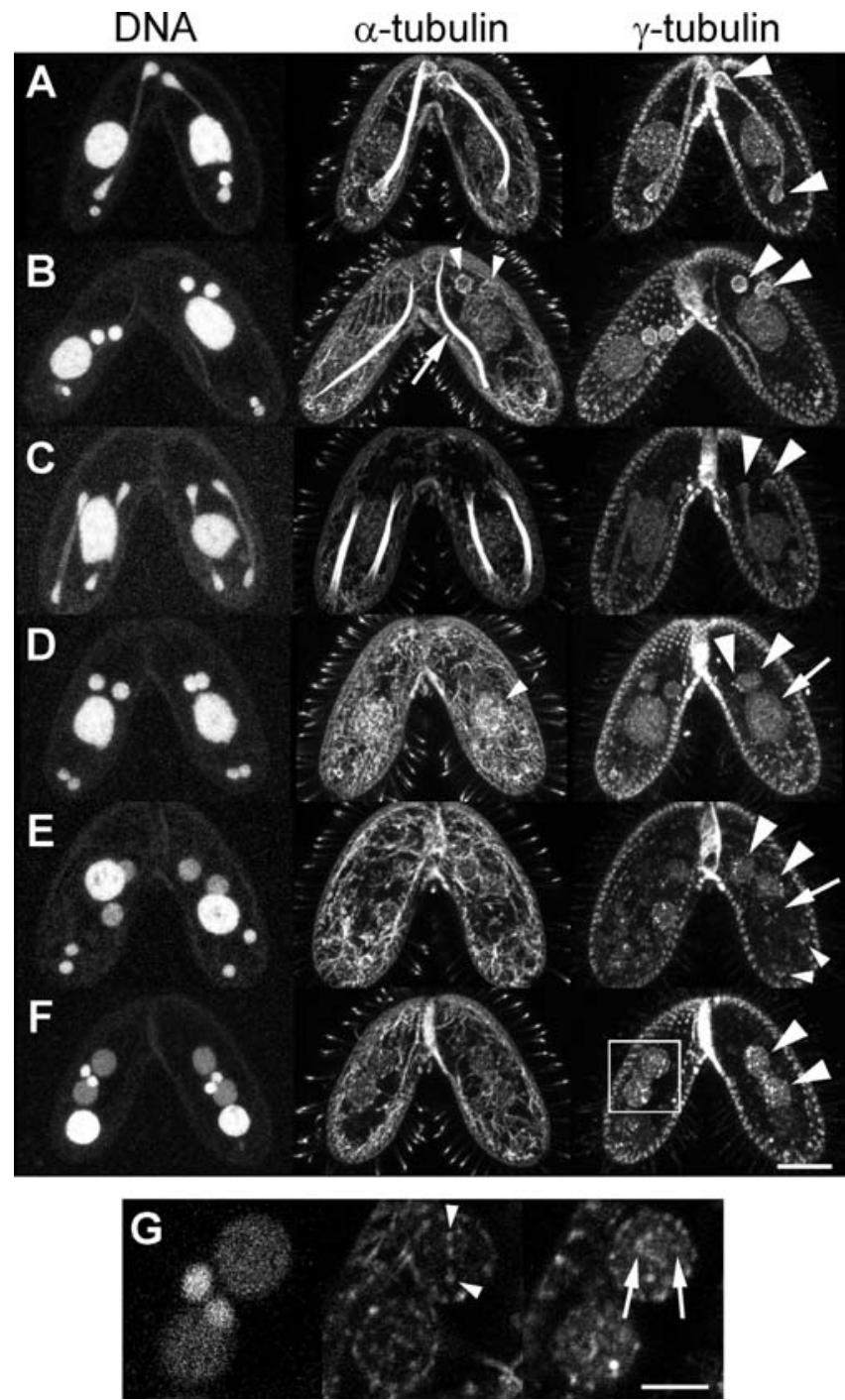

Fig. 4. Localization of DNA, MTs, and $\gamma$-tubulin during post-zygotic divisions and new MAC development. In all panels, $\alpha$-tubulin was used as a marker for MTs. (A) Late anaphase of first post-zygotic division. The two daughter nuclei were labeled with $\gamma$-tubulin (arrowheads). (B) After the first post-zygotic division. The two daughter nuclei (arrowheads) came closer to each other while the spindle MTs remained (arrow). Some MTs were formed around daughter nuclei (small arrowheads). (C) Anaphase of second post-zygotic division. The $\gamma$-tubulin signal was reduced (arrowheads). (D) After the second post-zygotic division. $\gamma$-tubulin was detectable in the new MACs (large arrowheads) and the parental MAC (arrow). Scattered MTs were formed in the parental MAC (small arrowhead). $\gamma$-tubulin started to accumulate at conjugation junction toward the separation of the pair. (E) Rearrangement of nuclear position. The $\gamma$-tubulin signal disappeared from the parental MAC (arrow) as the chromosomes condensed. New MACs (large arrowheads) and new MICs (small arrowheads) were labeled with $\gamma$-tubulin. (F) The characteristic pattern of nuclear position at the end of conjugation. The $\gamma$-tubulin signal in the new MACs (arrowheads) increased compared with the new MACs in E. Scale bar (A-F): $10 \mu \mathrm{m}$. See Supplementary Figure S2A for merged image. (G) Enlarged image of marked area in (F). Single focus image is shown. Some dotted MTs (arrowheads) in the new MACs colocalized with $\gamma$-tubulin, whereas some fraction of $\gamma$ tubulin did not colocalize with MTs (arrows). Scale bar (G): $4 \mu \mathrm{m}$. See Fig. S2B for merged image. 
During elongation of the crescent, the strong aggregation of $\gamma$-tubulin was remained (Fig. 1C, arrowhead), $\gamma$-tubulin dispersed all over the MT bundles. At the completion of crescent elongation, while some of them were released from there (Fig. 1D, right panel); the polarity of MTs consisting the fully-elongated crescent was thus not clear. The polarity can be one-directional or random.

\section{Bipolarity of meiotic spindle is established during short- ening of crescent \\ Corresponding to the condensation of MIC chromosome} (Fig. 1E, asterisk), an elongated crescent began to be shorten. MTs were quickly bundled at the chromosome-free region in the crescent (Fig. 1E, arrow), which suggests the presence of MT bundling protein in MIC. During shortening of the crescent, $\gamma$-tubulin gradually accumulated at both ends of crescent MT bundles (Fig. 1E, F, G, arrowheads). The accumulation was clearer when the length of the crescent became closer to the length of the meiotic spindle (Fig. $2 \mathrm{~A}, \mathrm{~B}$, arrowheads). This accumulation pattern of $\gamma$-tubulin during the crescent shortening suggests that the bipolarity of Tetrahymena meiotic spindle is established during shortening of the meiotic crescent. Previously, we analyzed a localization of $\gamma$-tubulin during vegetative growth (Kushida et al., 2011). Compared to mitotic spindle, the meiotic spindle seems to contain more $\gamma$-tubulin and MTs to form larger spindle, while both contains bundled MTs. Future comparisons of MT polarities in mitotic and meiotic spindles may contribute to the understanding of features of MT structures in each spindle.

\section{$\gamma$-tubulin accumulates on the selected gametic nucleus}

The selected post-meiotic nucleus was distinguished from other three nuclei by the selective accumulation of $\gamma$ tubulin (Fig. 3A, large arrowheads). Because radial MTs were specifically formed from this selected nucleus (Fig. 3A, arrows), we consider $\gamma$-tubulin is likely to aggregate on the outer surface of the selected nuclear envelope and induce polymerization of cytoplasmic MTs. Interestingly, the Tetrahymena citrate synthase, which forms a 14-nm filament in vitro (Numata et al., 1980, 1991; Takeda et al., 1997), shows preferential accumulation on the selected post-meiotic nucleus (Numata et al., 1985; Takagi et al., 1991) similarly to the MTs. Although the mechanism of nuclear fate determination is obscure, a preferential accumulation of $\gamma$ tubulin may play an important role.

\section{Possible roles of radial MTs in posterior-to-anterior migration and nuclear fusion}

The radial MTs, which were likely to be polymerized from the posterior pronucleus, elongated toward the destination of the pronucleus during posterior-to-anterior migration (Fig. 3D, arrow). Similar radial MTs were formed from the basket surrounding the anterior pronucleus (Fig. $3 \mathrm{H}$, arrow). These results suggest that the interaction between the radial MTs of the two pronuclei is important for posterior-to-anterior migration.

We observed that nucleus-associated $\gamma$-tubulin persisted even after fertilization (Fig. 3F, G, arrowhead). Both daughter nuclei of the first post-zygotic division were labeled with $\gamma$-tubulin (Fig. 4A, arrowheads). Because the two daughter nuclei approach each other after the first post-zygotic division (Fig. 4B, arrowheads; Cole and Sugai, 2012), accumulation of $\gamma$-tubulin may be required for movement of these daughter nuclei as well as the earlier pronuclear migration.

We were able to capture only a limited number of cells around the fertilization and during the post-zygotic divisions (Fig. 3D-E, 3G and 4A), suggesting that these processes progress so rapidly that intracellular structures are not rigidly fixed in most cells. Or MT structures might be very unstable and fragile during the period. This is the limitation of our immunofluorescence method, but inevitable because the method is optimized, and hence essential, for visualization of fine MT structures (Kushida et al., 2011). In the future, time-lapse imaging of tubulins $(\alpha / \beta$ and $\gamma)$ and nuclei will reveal more precisely their motions during the processes in living cells.

\section{Possible function of abundant cytoplasmic MTs formed after post-zygotic divisions}

We observed that the network of cytoplasmic MTs was highly augmented when the MT spindle of the second postzygotic division disappeared (Fig. 4D, middle panel), which was probably due to an accelerated MT polymerization with the increased concentration of free tubulin dimers. The massive polymerization of cytoplasmic MTs seemed to originate throughout the cell cortex rather than just from the surfaces of nuclei (Fig. 4D, middle panel).

By the end of the second post-zygotic division, two new MACs and two new MICs are differentiated. They move to characteristic positions relative to each other and the parental MAC: the two MICs are positioned between the two new MACs, and the parental MAC is positioned at the far posterior end of the cell (Cole and Sugai, 2012; Fig. 4F, left panel). This rearrangement of the new MACs and MICs and the parental MAC is more complex than the one-directional posterior-to-anterior migration of the pronucleus, because it includes multiple nuclear movements in different directions happening at the same time and place. Further analysis of MT-associated proteins is needed.

\section{Presence of $\gamma$-tubulin in swelling new MICs}

During the polymerization of abundant cytoplasmic MTs that occurs after second post-zygotic divisions, $\gamma$-tubulin enters the parental MAC (Fig. 4D, arrow), and scattered MTs formed in the parental MAC (Fig. 4D, small arrowhead). In addition, the distribution of $\gamma$-tubulin resembles the previously reported localization of MIC-derived small RNAs, which are required for new MAC development (Karrer, 2000, 2012; Schoeberl et al., 2012). With this exception, $\gamma$-tubulin enters the MAC only during $M$ phase of vegetative growth (Joachimiak et al., 2007; Kushida et al., 2011). The $\gamma$-tubulin in parental and developing MACs probably has an unknown conjugation-specific function.

\section{MTOC accumulation on conjugation junction}

The conjugation junction is formed at a boundary between two mating cells. EM studies proved the formation of hundreds of $0.1-0.2 \mu \mathrm{m}$ diameter pores as the result of membrane fusion events on the fusion plate (Wolfe, 1985; Cole, 2006). Indeed, the signal of $\gamma$-tubulin is detectable at the beginning of meiotic prophase in wildtype partner cell 
(Fig. 1A), because of transferring of $\mathrm{HA}$-tagged recombinant $\gamma$-tubulin protein from the other partner, cTTMGHA cell.

MTOC clearly accumulated on the junction at least three times during conjugation, namely during nuclear selection (Fig. 3A, small arrowhead), before and after pronuclear exchange (Fig. 3D, E) and just before pair separation (Fig. 4D-F). The conjugation junction is considered to play "a decisive role in selection of nuclei” (Frankel, 2000). In fact, the frequency of membrane fusion is highest near the selected nucleus (Cole, 2006), indicating close interaction between nuclei and the conjugation junction. $\gamma$-tubulin may be included within the 'electron dense matter' (Cole, 2006) on the surface of the junction, and distribute on selected nucleus as well (Fig. 3A, large arrowheads). During pronuclear exchange, EM studies report that an MT "basket" pushes the pronucleus against the conjugational junction
(Orias et al., 1983; Cole, 2006). $\gamma$-tubulin is closely associated with the basket (Fig. 3D, H), and clumps together just after pronuclear exchange (Fig. 3E, asterisk) to form 'plug at the post-exchange junction' (Cole, 2006). The accumulation of $\gamma$-tubulin on the junction is prominent during and after the development of new MAC and new MIC (Fig. 4D-F). These $\gamma$-tubulins are likely to be involved in the reconstruction of cell cortex on the conjugation junction that is required for pair separation.

In summary, the distribution of MTOC changes drastically following the proceeding of Tetrahymena developmental processes including meiosis, nuclear selection, nuclear exchange and new MAC development (Fig. 5). This study highlights the clear involvement of MT regulation in the modulation of germline nuclear dynamics indispensable for ciliate reproduction.
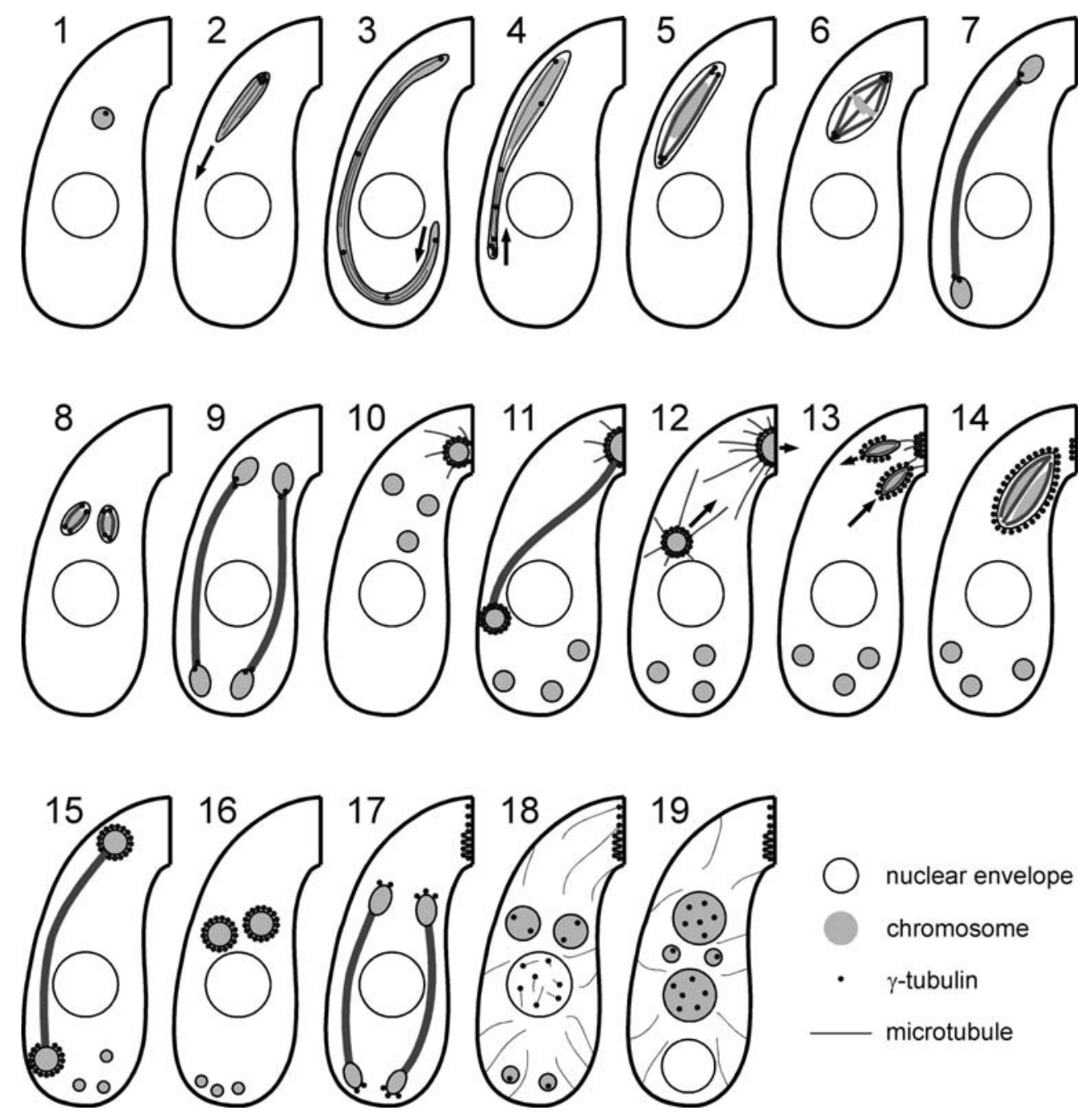

Fig. 5. Diagram showing the distribution of $\gamma$-tubulin during conjugation of Tetrahymena. For simplification, only one conjugant of pair is shown. Black solid line: nuclear envelope. Pale gray: chromosomes. Gray line: MT. Black dot: $\gamma$-tubulin. The chromosome of parental MAC is not present for simplification. 1) Early prophase of meiosis I. 2) The onset of crescent elongation. 3) Completion of crescent elongation. 4) Middle stage of crescent shortening. 5) Late stage of crescent shortening. 6) Metaphase, meiosis I. 7) Anaphase, meiosis I. 8) Metaphase, meiosis II. 9) Anaphase, meiosis II. 10) Nuclear selection. 11) Gametogenic mitosis. 12) Pronuclear exchange. 13) Before fertilization. 14) Synkaryon. 15) 1st postzygotic division. 16) Interphase after 1st postzygotic division. 17) 2nd postzygotic division. 18) Early development of new MAC. 19) Completion of the swelling of new MAC. 


\section{ACKNOWLEDGMENTS}

We are grateful to Prof. Martin A. Gorovsky (University of Rochester) for his kind donation of the Tetrahymena cell strain cTTMGHA. We are also grateful to Prof. Jacek Gaertig (University of Georgia) for his kind donation of the strains CU428 and B2086. This research was supported by a grant from the Sumitomo Foundation and from the Novartis Foundation to K. N.

\section{REFERENCES}

Allen S, Gibson I (1972) Genome amplification and gene expression in the ciliate macronucleus. Biochem Genet 6:293-313

Cole E (2006) The Tetrahymena conjugation junction. In "Cell-Cell Channels" Ed by F Baluska, D Volkmann, PW Barlow, Springer, New York, pp 39-62

Cole E, Sugai T (2012) Developmental progression of Tetrahymena through the cell cycle and conjugation. In "Methods in Cell Biology, Volume 109, Tetrahymena thermophila" Ed by K Collins, Academic Press, San Diego, pp 177-236

Frankel J (2000) Cell biology of Tetrahymena thermophila. In "Methods in Cell Biology, Volume 62, Tetrahymena thermophila" Ed by DJ Asai, JD Forney, Academic Press, San Diego, pp 127186

Gaertig J (2000) Molecular mechanisms of microtubular organelle assembly in Tetrahymena. J Eukaryot Microbiol 47: 185-190

Gaertig J, Fleury A (1992) Spatio-temporal reorganization of intracytoplasmic microtubules in associated with nuclear selection and differentiation during the developmental process in the ciliate Tetrahymena thermophila. Protoplasma 167: 74-87

Gönczy P, Pichler S, Kirkham M, Hyman AA (1999) Cytoplasmic dynein is required for distinct aspects of MTOC positioning, including centrosome separation, in the one cell stage Caenorhabditis elegans embryo. J Cell Biol 147: 135-150

Hamilton EP, Suhr-Jessen PB, Orias E (1988) Pronuclear fusion failure: an alternate conjugational pathway in Tetrahymena thermophila, induced by vinblastine. Genetics 118: 627-636

Joachimiak E, Pucciarelli S, Barchetta S, Ballarini P, Kaczanowska J, Miceli C (2007) Cell cycle-dependent expression of $\gamma$-tubulin in the amicronuclear ciliate Tetrahymena pyriformis. Protist 158: $39-50$

Kaczanowski A, Gaertig J, Kubiak J (1985) Effect of the antitubulin drug nocodazole on meiosis and post-meiotic development in Tetrahymena thermophila. Exp Cell Res 158: 244-256

Kaczanowski A, Ramel M, Kaczanowska J, Wheatley D (1991) Differentiation in conjugating pairs of Tetrahymena treated with the antitubulin drug nocodazole. Exp Cell Res 195: 330-337

Karrer KM (2000) Tetrahymena genetics: two nuclei are better than one. In "Methods in Cell Biology, Volume 62, Tetrahymena thermophila" Ed by DJ Asai, JD Forney, Academic Press, San Diego, pp 127-186

Karrer KM (2012) Nuclear dualism. In "Methods in Cell Biology, Volume 109, Tetrahymena thermophila" Ed by K Collins, Academic Press, San Diego, pp 29-52

Kushida Y, Nakano K, Numata O (2011) Amitosis requires $\gamma$-tubulinmediated microtubule assembly in Tetrahymena thermophila. Cytoskeleton 68: 89-96

Lindeman RE, Pelegri F (2012) Localized products of futile cycle/ Irmp promote centrosome-nucleus attachment in the zebrafish zygote. Curr Biol 22: 843-851

Loidl J, Scherthan H (2004) Organization and pairing of meiotic chromosomes in the ciliate Tetrahymena thermophila. J Cell Sci
117: $5791-5801$

Loidl J, Mochizuki K (2009) Tetrahymena meiotic nuclear reorganization is induced by a checkpoint kinase-dependent response to DNA damage. Mol Biol Cell 20: 2428-2437

Loidl J, Lukaszewicz A, Howard-Till RA, Koestler T (2012) The Tetrahymena meiotic chromosome bouquet is organized by centromeres and promotes interhomolog recombination. $\mathrm{J}$ Cell Sci 125: $5873-5880$

Malone CJ, Misner L, Le Bot N, Tsai MC, Campbell JM, Ahringer J, et al. (2003) The C. elegans Hook protein, ZYG-12, mediates the essential attachment between the centrosome and nucleus. Cell 115: 825-836

Martindale DW, Allis CD, Bruns PJ (1982) Conjugation in Tetrahymena thermophila. A temporal analysis of cytological stages. Exp Cell Res 140: 227-236

Minn IL, Rolls MM, Hanna-Rose W, Malone CJ. (2009) SUN-1 and ZYG-12, mediators of centrosome-nucleus attachment, are a functional SUN/KASH pair in Caenorhabditis elegans. Mol Biol Cell 20: 4586-4595

Numata O, Yasuda T, Hirabayashi T, Watanabe Y (1980) A new fiber-forming protein from Tetrahymena pyriformis. Exp Cell Res 129: 223-230

Numata O, Sugai T, Watanabe Y (1985) Control of germ cell nuclear behaviour at fertilization by Tetrahymena intermediate filament protein. Nature 314: 192-194

Numata O, Takemasa T, Takagi I, Hirono M, Hirano H, Chiba J, et al. (1991) Tetrahymena 14-nm filament-forming protein has citrate synthase activity. Biochem Biophys Res Commun 174: 1028-1034

Orias JD, Hamilton EP, Orias E (1983) A microtubule meshwork associated with gametic pronucleus transfer across a cell-cell junction. Science 222: 181-183

Ray C Jr (1956) Meiosis and nuclear behavior in Tetrahymena pyriformis. J Protozool 3: 88-96

Schoeberl UE, Kurth HM, Noto T, Mochizuki K (2012) Biased transcription and selective degradation of small RNAs shape the pattern of DNA elimination in Tetrahymena. Genes Dev 26 : 1729-1742

Shang Y, Li B, Gorovsky MA (2002) Tetrahymena thermophila contains a conventional $\gamma$-tubulin that is differentially required for the maintenance of different microtubule-organizing centers. $J$ Cell Biol 158: 1195-1206

Takagi I, Numata O, Watanabe Y (1991) Involvement of 14-nm filament-forming protein and tubulin in gametic pronuclear behavior during conjugation in Tetrahymena. J Protozool 38: 345-351

Takeda T, Watanabe Y, Numata O (1997) Direct demonstration of the bifunctional property of Tetrahymena 14-nm filament protein/citrate synthase following expression of the gene in Escherichia coli. Biochem Biophys Res Commun 237: 205-210

Wloga D, Frankel J (2012) From molecules to morphology: cellular organization of Tetrahymena thermophila. In "Methods in Cell Biology, Volume 109, Tetrahymena thermophila" Ed by K Collins, Academic Press, San Diego, pp 83-140

Wolfe J (1985) Cytoskeletal reorganization and plasma membrane fusion in conjugating Tetrahymena. J Cell Sci 73: 69-85

Wolfe J, Hunter B, Adair WS (1976) A cytological study of micronuclear elongation during conjugation in Tetrahymena. Chromosoma 55: 289-308

(Received August 17, 2014 / Accepted September 5, 2014) 